\title{
Effectiveness of Osteopathic Manipulative Medicine vs Concussion Education in Treating Student Athletes With Acute Concussion Symptoms
}

Sheldon C. Yao, DO; Hallie Zwibel, DO; Nicole Angelo, OMS IV; Adena Leder, DO; Jayme Mancini, DO, PhD

From Departments of Osteopathic Manipulative Medicine (Drs Yao, Leder, and Mancini) and Family Medicine (Dr Zwibel) at the New York Institute of Technology College of Osteopathic Medicine (Student Doctor Angelo). This article was completed as part of the requirement toward Dr Yao earning the designation of Fellow in the American Academy of Osteopathy.

Financial Disclosures: None reported.

Support: None reported.

Address correspondence to Sheldon C. Yao, DO, New York Institute of Technology College of Osteopathic Medicine,

Department of Osteopathic Manipulative Medicine, $\mathrm{PO}$ Box 8000 Northern Blvd, Serota Building Room 127 Old Westbury, NY 11568-8000.

Email: sheldon.yao@nyit.edu Submitted March 22, 2019; accepted May 15, 2019.
Context: Concussion, a type of mild traumatic brain injury, is a disruption in normal brain function due to head injury. New-onset symptoms from concussion vary, likely depending on the areas of the head and neck affected; they can be severe and debilitating. Current treatment options are limited and difficult to individualize. Osteopathic manipulative medicine (OMM) can aid musculoskeletal restrictions that can potentially improve concussion symptoms.

Objective: To assess concussion symptom number and severity in participants with concussion who received either OMM or an educational intervention.

Methods: A randomized controlled trial was conducted at the New York Institute of Technology Academic Health Care Center. Patients presenting to the center with concussion-like symptoms due to recent head injury within the previous 7 days provided consent and were randomized into 2 intervention groups, receiving either 1 OMM treatment $(n=16)$ or 1 concussion education intervention $(n=15)$. Participants were assessed before and after the intervention with the validated Symptom Concussion Assessment Tool fifth edition (SCAT-5) for quantification of number of symptoms and their severity. Collected data were analyzed using the Mann-Whitney $U$ test and the repeated-measures analysis of variance.

Results: Thirty-one participants were enrolled in the study; after 1 control participant was excluded due to incomplete data, 30 patient records were analyzed. The OMM intervention group had a significant decrease in symptom number $(P=.002)$ and symptom severity $(P=.001)$ compared with the concussion education group.

Conclusion: When used in the acute setting, OMM significantly decreased concussion symptom number and severity compared with concussion education. This study demonstrates that integration of OMM using a physical examination-guided, individualized approach is safe and effective in the management of new-onset symptoms of uncomplicated concussions. (Clinicaltrials.gov No. NCT02750566)

J Am Osteopath Assoc. 2020;120(9):607-614. Published online August 7, 2020. doi:10.7556/jaoa.2020.099

Keywords: athletes, concussion, osteopathic manipulative medicine raumatic brain injury (TBI) is an injury to the head that interferes with normal brain function. The Centers for Disease Control and Prevention estimate that 1.7 million TBIs occur annually within the United States, with 300,000 of those being sports-related. ${ }^{1,2}$ Other studies have suggested that this number only accounts 
for sports-related TBIs that involve loss of consciousness and more accurate estimates range from 1.7 to 3.8 million per year. ${ }^{2}$ The incidence of TBI caused by repetitive injury not involving loss of consciousness probably results in additional incidents of undiagnosed concussion, which is a mild $\mathrm{TBI}^{3}$

Concussions are caused by a blow to the head or other impulsive force to the body transmitted to the head, thereby causing an immediate and generally irreversible primary injury due to mechanical damage imposed by acceleration and deceleration forces. ${ }^{4}$ The secondary injury phase, occurring at the cellular level, includes neuronal cell membrane disruption and axonal stretch. ${ }^{5-8}$ This phase alters the membrane potential, causing ion and neurotransmitter redistribution that leads to increases in oxidative stress levels, which in turn may contribute to the manifestation of concussion symptoms. ${ }^{4-8}$ Further release of free radicals, including reactive oxygen species and purines, causes a downstream inflammatory reaction in the meninges and brain parenchyma, potentiating concussion symptoms over time. ${ }^{6-9}$ Because the signs and symptoms can clinically manifest anywhere from minutes to hours after injury, concussion can be difficult to diagnose even for licensed health care professionals. ${ }^{4}$ Symptoms can include somatic manifestations such as headaches, cognitive and emotional fluctuations, impaired balance, decreased coordination, and vertigo. ${ }^{4,10}$

Current methods for concussion diagnosis and assessment include a physical examination for signs of trauma and neurologic changes, neurocognitive testing, and symptom checklists. Self-reported symptom inventories have been shown to have higher sensitivity for detecting concussion than written neurocognitive examinations and balance testing. ${ }^{11}$ The Symptom Concussion Assessment Tool fifth edition (SCAT-5) includes a self-reported questionnaire to quantify the number of symptoms and their severity.

Because of the variance in the regions of the head and neck affected by a concussion, a universal treatment model is inappropriate. ${ }^{12}$ The current American
Academy of Neurology practice parameters recommend rest until the injured patient is asymptomatic, followed by a gradual return-to-play progression. ${ }^{13,14} \mathrm{~A}$ recent large systematic review ${ }^{15}$ of concussion management protocols found that recommendations for rest are broad and difficult to translate to individual cases. Other studies ${ }^{16,17}$ have shown significant variability in the benefits of rest, as well as indications for exercisebased programs, in concussion management.

With the discovery of the dural lymphatic vessels, termed glymphatics, osteopathic manipulative medicine (OMM) models can be applied to affect the drainage of this system. ${ }^{18,19}$ OMM involves a detailed physical examination to evaluate for specific somatic dysfunctions that guide the osteopathic treatment approach. In general, forces induced by $\mathrm{OMM}$ are directed to reduce dysfunction of the neuromusculoskeletal system and its connective fascia, thereby improving the natural physiologic function. In cases of concussion, a major goal of the techniques is to enhance circulation of lymphatic drainage. ${ }^{20}$ By aiding in the clearance of substances such as oxidative mediators, toxic metabolic waste, and cellular debris carried within the glymphatic system or cerebrospinal fluid, OMM could potentially decrease the severity and duration of concussion-related symptoms. ${ }^{18,19}$ Previous studies $^{21,22}$ have found OMM to be effective in improving symptoms, including dizziness, nausea, tinnitus, and imbalance. The aim of this study was to assess concussion symptom number and severity in participants who received either OMM or an educational intervention to determine the role of $\mathrm{OMM}$ in the management of concussion.

\section{Methods}

This randomized, controlled trial was approved by the New York Institute of Technology (NYIT) institutional review board and registered at clinicaltrials.gov (NCT02750566). It was conducted at the NYIT Academic Health Care Center (AHCC). 


\section{Recruitment}

Patients were notified of the study by the treating sports medicine physician/neurologist (H.Z., A.L.) when they presented to the clinic for concussion symptom evaluation. Many were student athletes who were directed to come for clearance for return to play. From November 2015 to February 2018, men and women between 18 and 50 years of age with a diagnosis of concussion (International Classification of Diseases, Ninth Revision and 10th Revision) within 2 weeks of the event leading to head injury who were determined to be clear of any life-threatening or emergent conditions by a physician were included in this study. Patients were excluded if they had a current or previous diagnosis of a neurodegenerative condition or spinal cord injury that might confound results, an inability to complete assessment tools, absolute contraindications to OMM (eg, skull fracture, signs of intracranial bleeding, cervical fracture, dissection, or stroke), pregnancy, postinjury related loss of consciousness for greater than 2 minutes, seizures, intractable vomiting, or paralysis as self-reported or witnessed at the time of the event that led to concussion. Participants were current or newly referred patients to the AHCC concussion program, including student athletes attending NYIT or other surrounding universities.

\section{Participants}

Patients who presented to the NYIT-AHCC with a history of recent head-related injury and new onset neurologic symptoms including, but not limited to, headache, amnesia, visual disturbance, nausea, dizziness, balance disturbance, sleep disturbance, photophobia, phonophobia, fogginess, lethargy, and tinnitus and received a diagnosis of concussion were notified of the current study and invited to participate. On the first visit after eligibility assessment and screening questionnaires, enrolled patients who provided informed consent were given a hard copy of the SCAT5 questionnaire to complete. Then they were randomly assigned by blocks of 2 into 1 of 2 intervention groups: OMM intervention or education. Participants placed in the OMM interven- tion group received an osteopathic structural examination and a 30-minute osteopathic treatment session. Participants in the concussion education intervention group received a 30-minute concussion education session. Participants followed up 48 to 72 hours later (Visit 2) and completed the SCAT5 questionnaire.

\section{OMM Intervention Group}

This intervention had 3 parts. Participants underwent an osteopathic structural examination and 1 subsequent 30-minute OMM session, both of which were performed by a board-certified neuromusculoskeletal medicine/OMM physician (J.M., S.Y.). Part 1 of the protocol included the osteopathic structural examination and assessment for somatic dysfunctions - specifically, examination of the cranium included cranial sutures, the occipitoatlantal joint, the occipitomastoid joint, cranial strain patterns, and the cranial rhythmic impulse. The spinal examination included cervical, thoracic, lumbar, and sacral regions. Examination of the thoracic cage included the superior thoracic aperture, sternal and clavicular areas, thoracic diaphragm, and rib excursion function. Examination of the pelvis and extremities was also performed. In part 2 of the protocol, OMM treatment guidelines were created based on individual osteopathic structural examination findings pertinent to the mechanism of injury. Part 3 comprised the OMM techniques, with the primary goal of improving circulation. Specific techniques were performed on all participants to improve glymphatic and lymphatic drainage of the head and neck; OMM technique was standardized among treating physicians.

The lymphatic drainage techniques performed included thoracic inlet release to alleviate musculoskeletal and fascial restrictions to drainage, rib raising to improve autonomic nervous system balance and control of circulation, occipitoatlantal decompression, $\mathrm{V}$ spread of the occipitomastoid sutures, balanced membranous tension for cranial strain patterns, cranial lifts to remove restrictions of the cranial bones and dural venous sinus drainage. All participants received 
compression of the fourth ventricle at the end of cranial treatment. OMM options to appropriately address somatic dysfunctions included balanced ligamentous tension, muscle energy techniques, facilitated positional release, articulatory techniques, high-velocity, low-amplitude, and counterstrain. Osteopathic structural examination findings and OMM techniques were documented by all physicians performing the intervention.

\section{Concussion Education Intervention Group}

The education intervention consisted of a physician-led, 30-minute concussion education session controlling for placebo effects of the face-to-face time spent with a physician that the OMM intervention group had. This session included topics from the Centers for Disease Control and Prevention publications "Facts About Concussion and Brain Injury: Where to Get Help" and "Heads Up Concussion: A Fact Sheet for Teachers, Counselors, and School Professionals." Concussion education included physician-led education on the definition of concussion and how to recognize the cognitive, behavioral, and physical signs of concussion. Protocol also included discussion of diagnosis, risk factors, and predictors of concussion, as well as recommendations for recovery/management and expectations for return to play/work. Concussion education topics discussed, questions addressed, and comments/observations were documented by all physicians performing the intervention.

\section{Measuring Parameters}

SCAT5 includes a self-reported symptom evaluation portion, an objective cognitive portion, and a physical examination portion. This study used the symptom evaluation portion, which includes 22 concussion symptoms rated by participants on a 7-point Likert scale ranging from 0 to 6 . The completed symptom evaluation generates a symptom number with a range from 0 (no symptoms) to 22 (all symptoms). A symptom severity score is also generated, with a maximum possible score of 132 (Figure).

\section{Statistical Analysis}

Quantitative measurement via SCAT5 preintervention symptom number and severity values were compared between the groups using independent sample $t$ tests. Mean differences between postintervention and preintervention symptom number and severity values were calculated using the Mann-Whitney $U$ test. Qualitative measures of medical history and history of present illness were documented and evaluated for potential preintervention differences in concussion subtypes and confounding variables.

\section{Results}

Of 73 participants informed of the study and screened for eligibility, 42 did not qualify or declined to participate in the study. A total of 31 participants (aged 18-27 years [mean, 19.9 years]; 19 men [61.3\%] and 12 women [38.7\%]) completed the study; however, 1 control participant had to be excluded from the analysis because of incomplete data. Time between head injury and enrollment in the study ranged from 0 to 12 days, with a mean of 3.43 days.

In comparing preintervention differences in concussion symptom number and severity values between groups, there was no significant difference in preintervention mean symptom numbers between the OMM intervention group $(\mathrm{n}=16$; mean [SD], 14.38 [5.04]) and the concussion education intervention group (n=14; mean [SD], 13.07 [5.00]; $P=.615$ ). No significant difference was found in initial symptom severities between the OMM group (mean [SD], 38.31 [22.20]) and the control group (mean [SD], 31.00 [16.03]; $P=.196$ ).

The mean improvement from before to after the interventions was significantly different between groups in both symptom number and severity values. The OMM intervention group had a significantly greater decrease in the mean (SD) number of symptoms $(-3.93$ [3.92]) than the concussion education-intervention group ( -0.64 [0.97]); $P=.002$. The OMM intervention group was also found to have a significantly greater 


\section{SYMPTOM EVALUATION}

\section{How do you feel?}

\begin{tabular}{|c|c|c|c|c|c|c|c|}
\hline \multirow[b]{2}{*}{ Headache } & \multirow{2}{*}{$\begin{array}{c}\text { None } \\
0 \\
\end{array}$} & \multicolumn{2}{|c|}{ Mild } & \multicolumn{2}{|c|}{ Moderate } & \multicolumn{2}{|c|}{ Severe } \\
\hline & & 1 & 2 & 3 & 4 & 5 & 6 \\
\hline "Pressure in head" & 0 & 1 & 2 & 3 & 4 & 5 & 6 \\
\hline Neck Pain & 0 & 1 & 2 & 3 & 4 & 5 & 6 \\
\hline Nausea or vomiting & 0 & 1 & 2 & 3 & 4 & 5 & 6 \\
\hline Dizziness & 0 & 1 & 2 & 3 & 4 & 5 & 6 \\
\hline Blurred vision & 0 & 1 & 2 & 3 & 4 & 5 & 6 \\
\hline Balance problems & 0 & 1 & 2 & 3 & 4 & 5 & 6 \\
\hline Sensitivity to light & 0 & 1 & 2 & 3 & 4 & 5 & 6 \\
\hline Sensitivity to noise & 0 & 1 & 2 & 3 & 4 & 5 & 6 \\
\hline Feeling slowed down & 0 & 1 & 2 & 3 & 4 & 5 & 6 \\
\hline Feeling like "in a fog" & 0 & 1 & 2 & 3 & 4 & 5 & 6 \\
\hline "Don't feel right" & 0 & 1 & 2 & 3 & 4 & 5 & 6 \\
\hline Difficulty concentrating & 0 & 1 & 2 & 3 & 4 & 5 & $\epsilon$ \\
\hline Difficulty remembering & 0 & 1 & 2 & 3 & 4 & 5 & 6 \\
\hline Fatigue or low energy & 0 & 1 & 2 & 3 & 4 & 5 & 6 \\
\hline Confusion & 0 & 1 & 2 & 3 & 4 & 5 & 6 \\
\hline Drowsiness & 0 & 1 & 2 & 3 & 4 & 5 & 6 \\
\hline Trouble falling asleep & 0 & 1 & 2 & 3 & 4 & 5 & 6 \\
\hline More emotional & 0 & 1 & 2 & 3 & 4 & 5 & 6 \\
\hline Irritability & 0 & 1 & 2 & 3 & 4 & 5 & 6 \\
\hline Sadness & 0 & 1 & 2 & 3 & 4 & 5 & 6 \\
\hline Nervous or Anxious & 0 & 1 & 2 & 3 & 4 & 5 & 6 \\
\hline
\end{tabular}

Total number of symptoms (Maximum possible 22)

Symptom severity score (Maximum possible 132)

Do the symptoms get worse with physical activity? Yes No Do the symptoms get worse with mental activity? Yes No Self rated Self rated and clinician monitored

Clinician interview Self rated with parent input

Overall rating: If you know the athlete well prior to the injury, how different is the athlete acting compared to his/her usual self.

Please circle one response:

\begin{tabular}{|l|l|l|l|} 
No different & Very different & Unsure & N/A \\
\hline
\end{tabular}

Figure.

Sports concussion assessment tool, 5th Edition Symptom Evaluation. Reprinted with permission of BMJ Publishing Group Ltd.

decrease in mean symptom severity $(-17.33$ [17.38])

than the concussion education group $(-3.64 ;[5.89])$ $(P=.001)$.

\section{Discussion}

The treatment of patients with concussion who have persistent symptoms has previously been reported to vary significantly. ${ }^{23}$ In the current study, the SCAT5 questionnaire quantified information from the history of the present illness and a review of systems that are typically assessed qualitatively. Among adult patients with concussions, those with a higher symptom burden after injury seem to have prolonged symptoms. ${ }^{24}$ Concussion can result in a wide range of symptoms that can be challenging for physicians to address. 
Although OMM has previously been reported in case series and prospective reports of low sample size with or without controls to be safe and to improve symptoms, the current study demonstrates that OMM may be performed safely. The findings in this study support the application of OMM to reduce the number of concussion symptoms and their severity.

Several concepts may explain the positive effects of OMM in our study. It has been calculated that concussion injuries can be caused by forces between 50 to $250 \mathrm{~g}{ }^{25}$ These impacts can result in musculoskeletal restrictions and physiologic changes that can contribute to concussion symptoms. Cranial strain patterns from participants in the OMM arm of our study demonstrated an increased trend toward non-physiologic strains. ${ }^{26}$ Studies have shown that patients with neck pain have demonstrated altered proprioception, balance disturbances, altered eye movement control, and altered postural activity of cervical muscles. ${ }^{27,28} \mathrm{OMM}$ with a biomechanical treatment approach can potentially reduce musculoskeletal restrictions that can contribute to concussion symptoms such as headaches. OMM has shown benefit in patients with migraine, as well as other types of headaches. ${ }^{29}$ Manual techniques have also been demonstrated to be beneficial in treating cervicogenic headache, which can occur after concussion and often presents with pain and decreased mobility of the neck. ${ }^{30,31}$

The secondary phase of injury that occurs after concussion results in the formation of reactive metabolites, which are proposed to cause a downstream inflammatory reaction and perpetuate the presence of concussion symptoms. ${ }^{6-9}$ Manual techniques are theorized to stimulate the lymphatic system via circulation, aid in the removal of biochemical waste, augment body fluid dynamics, and decrease sympathetic nervous system activation. Specifically, a previous study demonstrated the efficacy of manual techniques in resolving increased serum enzyme levels in sports medicine and rehabilitation settings. ${ }^{32}$ With the discovery of the glymphatic system and its similarities to the body's lymphatic system, manual techniques have the potential to address these structures. Studies have found OMM to be effective in promoting lymphatic drainage, thereby providing further evidence for its potential to be applied to the glymphatics. ${ }^{33,34}$ Sleep has also been documented to enhance the efficacy and flow of the glymphatic system. ${ }^{35}$ The OMT arm of the present study demonstrated improved self-reported sleep quality when compared to the control arm. ${ }^{36}$ Because of the alterations in blood flow and glucose metabolism after concussion, a possible role for OMM in augmenting clearance of inflammatory mediators, causing the decrease in acute symptoms, can be theorized.

Dysfunction in the autonomic system has been found to be a major factor in the symptoms of concussion. ${ }^{37}$ OMM studies have demonstrated the positive effects of osteopathic techniques in affecting autonomic measures such as salivary immunoglobulin A levels in stressed participants and heart rate variability. ${ }^{38,39}$ In addition to balancing autonomics, OMM can also potentially decrease allostatic load and assist patients in healing from their injuries. ${ }^{40}$

There are significant limitations in this study, including a small sample size and a narrow participant population of otherwise healthy young student athletes. Increasing the participant number will further investigate the effects of OMM on concussion symptoms. Most participants had mild concussions and were treated within 1 week of injury. This study did not investigate chronic and more severe head injury cases. SCAT5 provided a sensitive tool for assessing symptom presence and progression; however, additional objective tools can be used to better study the effects of OMM. The long-term effects of OMM beyond a single treatment should be considered. The OMM provided to participants followed a general guideline approach but varied in applied techniques and regions treated specific to the participant's presentation and the skill set of the physician. With additional data, concussion specific structural findings and efficacy of specific treatment modalities can potentially be elucidated. 


\section{Conclusion}

When used in the acute setting, OMM significantly decreased concussion symptom number and severity compared with concussion education. This study demonstrates that integration of OMM using a physical examination-guided individualized approach is safe and effective in the management of new-onset symptoms of uncomplicated concussions. Additional research will help to establish the effectiveness of OMM in the management of concussion symptoms.

\section{Author Contributions}

All authors provided substantial contributions to conception and design, acquisition of data, or analysis and interpretation of data; all authors drafted the article or revised it critically for important intellectual content; all authors gave final approval of the version of the article to be published; and all authors agree to be accountable for all aspects of the work in ensuring that questions related to the accuracy or integrity of any part of the work are appropriately investigated and resolved.

\section{References}

1. Faul M, Xu L, Wald MM, Coronado VG. Traumatic Brain Injury in the United States: Emergency Department Visits, Hospitalizations and Deaths 2002-2006. Centers for Disease Control and Prevention; 2010.

2. Langlois JA, Rutland-Brown W, Wald MM. The epidemiology and impact of traumatic brain injury: a brief overview. J Head Trauma Rehabil. 2006;21(5):375-378. doi:10.1097/00001199200609000-00001

3. Meehan WP III, Mannix RC, O'Brien MJ, Collins MW. The prevalence of undiagnosed concussions in athletes. Clin J Sport Med. 2013;23 (5):339-342. doi:10.1097/JSM.0b013e318291d3b3

4. McCrory P, Meeuwisse WH, Aubry M, et al. Consensus statement on concussion in sport: the 4th International Conference on Concussion in Sport held in Zurich, November 2012. Br J Sports Med. 2013;47:250-258. doi: 10.1136/bjsports-2013-092313

5. Mechtler LL, Shastri KK, Crutchfield KE. Advanced neuroimaging of mild traumatic brain injury. Neurol Clin. 2014;32(1):31-58. doi:10.1016/ j.ncl.2013.08.002

6. Giza CC, Hovda DA. The neurometabolic cascade of concussion. $J$ Athl Train. 2001;36(3):228-235.

7. Prins M, Greco T, Alexander D, Giza CC. The pathophysiology of traumatic brain injury at a glance. Dis Model Mech. 2013;6 (6):1307-1315. doi:10.1242/dmm.011585

8. McKee AC, Daneshvar DH. The neuropathology of traumatic brain injury. Handb Clin Neurol. 2015;127:45-66. doi:10.1016/ B978-0-444-52892-6.00004-0

9. Deshpande LS, Sun DA, Sombati S, et al. Alterations in neuronal calcium levels are associated with cognitive deficits after traumatic brain injury. Neurosci Lett. 2008;441(1):115-119. doi:10.1016/j.neulet.2008.05.113
10. Traumatic brain injury \& concussion. Centers for Disease Control and Prevention website. https://www.cdc.gov/traumaticbraininjury/index. html. Accessed July 20, 2017

11. Broglio SP, Macciocchi SN, Ferrara MS. Sensitivity of the concussion assessment battery. Neurosurgery. 2007;60(6):1050-1057. doi:10.1227/01.NEU.0000255479.90999.C0

12. Stewart GW, McQueen-Borden E, Bell RA, Barr T, Juengling J Comprehensive assessment and management of athletes with sport concussion. Int J Sports Phys Ther. 2012;7(4):433-447.

13. Giza CC, Kutcher JS, Ashwal S, et al. Summary of evidence-based guideline update: evaluation and management of concussion in sports: report of the Guideline Development Subcommittee of the American Academy of Neurology. Neurology. 2013;80(24):2250-2257. doi:10.1212/WNL.0b013e31828d57dd

14. Howell DR, Mannix RC, Quinn B, Taylor JA, Tan CO, Meehan WP III. Physical activity level and symptom duration are not associated after concussion. Am J Sports Med. 2016;44(4):1040-1046. doi:10.1177/ 0363546515625045

15. Valovich McLeod TC, Lewis JH, Whelihan K, Welch Bacon CE. Rest and return to activity after sport-related concussion: a systematic review of the literature. J Athl Train. 2017;52(3):262-287. doi:10.4085/ 1052-6050-51.6.06

16. Brolinson PG. Management of sport-related concussion: a review. Clin J Sport Med. 2014;24(1):89-90. doi:10.1097/ JSM.0000000000000062

17. Schneider KJ, Iverson GL, Emery CA, McCrory P, Herring SA Meeuwisse WH. The effects of rest and treatment following sport-related concussion: a systematic review of the literature. Br J Sports Med. 2013;47(5):304-307. doi:10.1136/bjsports-2013-092190

18. Louveau A, Smirnov I, Keyes TJ, et al. Structural and functional features of central nervous system lymphatic vessels. Nature. 2015;523(7560):337-341. doi:10.1038/nature14432

19. Hitscherich K, Smith K, Cuoco JA, et al. The glymphatic-lymphatic continuum: opportunities for osteopathic manipulative medicine. J Am Osteopath Assoc. 2016;116:170-177. doi:10.7556/jaoa.2016.033

20. McCombs TM. OMT: evidence, research, and practice. J Am Osteopath Assoc. 2006;106:379-380.

21. Chappell C, Dodge E, Dogbey GY. Assessing the immediate effect of osteopathic manipulation on sports related concussion symptoms. Osteopath Fam Phys. 2015;7(4):30-35.

22. Guernsey DT III, Leder A, Yao S. Resolution of concussion symptoms after osteopathic manipulative treatment: a case report. J Am Osteopath Assoc. 2016;116(3):e13-e17. doi:10.7556/jaoa.2016.036

23. Rose SC, Fischer AN, Heyer GL. Physicians' management practices and perceived health risks when postconcussion symptoms persist. Sports Health. 2016;8(1):37-42. doi:10.1177/1941738115591948

24. Meehan WP III, O'Brien MJ, Geminiani E, Mannix R. Initial symptom burden predicts duration of symptoms after concussion. J Sci Med Sport. 2016;19(9):722-725. doi:10.1016/j.jsams.2015.12.002

25. Leddy JJ, Baker JG, Merchant A, et al. Brain of strain: symptoms alone do not distinguish physiologic concussion from cervical/ vestibular injury. Clin J Sport Med. 2015;25(3):237-242. doi:10.1097/ JSM.0000000000000128

26. Schwartzberg L, Aslanyan L, Angelo N, et al. Cranial strain patterns associated with concussions. J Am Osteopath Assoc. 2020;120(9): In Press. doi:10.7556/jaoa.2020.098 
27. Post A, Hoshizaki TB, Zemek R, et al. Pediatric concussion: biomechanical differences between outcomes of transient and persistent (> 4 weeks) postconcussion symptoms. J Neurosurg Pediatr. 2017;19(6):641-651. doi:10.3171/2016.11.PEDS16383

28. Jull G, Falla D, Treleaven J, Hodges P, Vicenzino B. Retraining cervical joint position sense: the effect of two exercise regimes. J Orthop Res. 2007;25(3):404-412. doi:10.1002/jor.20220

29. Cerritelli F, Lacorte E, Ruffini N, Vanacore N. Osteopathy for primary headache patients: a systematic review. J Pain Res. 2017;10:601-611. doi:10.2147/JPR.S130501

30. Garcia JD, Arnold S, Tetley K, Voight K, Frank RA. Mobilization and manipulation of the cervical spine in patients with cervicogenic headache: any scientific evidence? Front Neurol. 2016;7:40. doi:10.3389/fneur.2016.00040

31. Morin $M$, Langevin $P$, Fait $P$. Cervical spine involvement in mild traumatic brain injury: a review. J Sports Med. 2016;2016:1590161. doi:10.1155/2016/1590161

32. Vairo GL, Miller SJ, McBrier NM, Buckley WE. Systematic review of efficacy for manual lymphatic drainage techniques in sports medicine and rehabilitation: an evidence-based practice approach. J Man Manip Ther. 2009;17(3):e80-e89. doi:10.1179/jmt.2009.17.3.80E

33. Majewski-Schrage T, Snyder K. The effectiveness of manual lymphatic drainage in patients with orthopedic injuries. J Sport Rehabil. 2016;25 (1):91-97. doi:10.1123/jsr.2014-0222
34. Lee-Wong M, Karagic M, Doshi A, Gomez S, Resnick D. An osteopathic approach to chronic sinusitis. J Aller Ther. 2011;2:109. doi:10.4172/2155-6121.1000109

35. Benveniste $\mathrm{H}$, Liu X, Koundal S, et al. The glymphatic system and waste clearance with brain aging: a review. Gerontology. 2019;65(2):106-119. doi:10.1159/000490349

36. Mazzeo S, Silverberg C, Oommen T, et al. Effects of osteopathic manipulative treatment on sleep quality in student athletes after concussion: a pilot study. J Am Osteopath Assoc. 2020;120(9): In Press. doi:10.7556/jaoa.2020.100

37. McCorry LK. Physiology of the autonomic nervous system. Am J Pharm Educ. 2007;71(4):78. doi:10.5688/aj710478

38. Giles PD, Hensel KL, Pacchia CF, Smith ML. Suboccipital decompression enhances heart rate variability indices of cardiac control in healthy participants. J Altern Complement Med. 2013;19 (2):92-96. doi:10.1089/acm.2011.0031

39. Saggio G, Docimo S, Pilc J, Norton J, Gilliar W. Impact of osteopathic manipulative treatment on secretory immunoglobulin a levels in a stressed population. J Am Osteopath Assoc. 2011;111(3):143-147.

40. Lunghi $C$, Tozzi P, Fusco G. The biomechanical model in manual therapy: is there an ongoing crisis or just the need to revise the underlying concept and application? J Bodyw Mov Ther. 2016;20 (4):784-799. doi:10.1016/j.jbmt.2016.01.004

๑) 2020 American Osteopathic Association 